\title{
Über Medizingeschichte als Schweizergeschichte
}

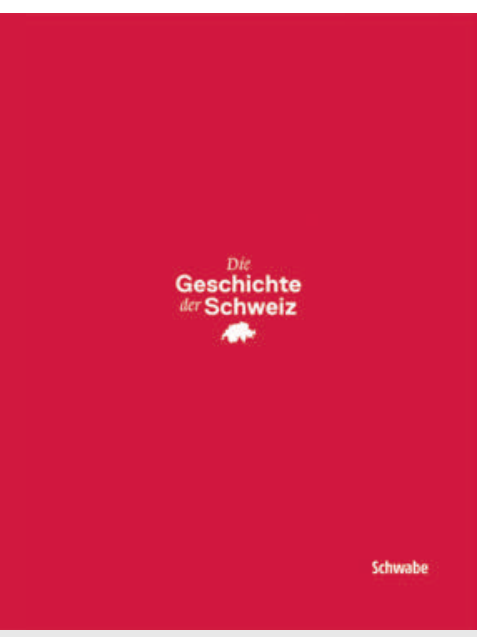

Ein repräsentativer Gesamtüberblick von den Pfahlbauern bis zum SwissGrounding.

* PD Dr. rer. soc. Eberhard Wolff ist Kulturwissenschaft ler, Medizinhistoriker und Mitglied der Redaktion Medizingeschichte der Schweizerischen Ärztezeitung.
Im Frühjahr 1963 brachte eine Typhusepidemie den Kurort Zermatt in die internationalen Schlagzeilen und für einige Zeit um seinen Ruf als Gesundbrunnen. Drei Menschen starben, 450 wurden hospitalisiert. Dazu kam ein immenses Image-Desaster. Wer war verantwortlich? Hatte ein italienischer Saisonnier die Seuche eingeschleppt, wie es zunächst hiess? Oder waren es das rückständige Kanalisationssystem und die Vertuschungshaltung der einheimischen Behörden?

An die kleine, aber wichtige Episode wird auf Seite 566 des fünfpfündigen Überblickswerks erinnert, um das es hier gehen soll: die kürzlich im Schwabe-Verlag erschienene deutschsprachige «Geschichte der Schweiz» [1]. Ein mutiger und repräsentativer Gesamtüberblick von den Pfahlbauern bis zum Swissair-Grounding, ein lesefreundliches Nachschlagewerk und solides Lesebuch.

Die Zermatter Begebenheit liefert dabei eine kleine Vignette für das Epochenkapitel, in dem der Herausgeber Georg Kreis die jüngste Geschichte der Schweiz auf dem Weg in die Gegenwart skizziert. «Viel Zukunft - erodierende Gemeinsamkeit» ist der Titel. Im Zentrum stehen das enorme Wirtschaftswachstum und der grundlegende kulturelle Wandel. Kreis sieht die Epoche noch als eine «historiographische Baustelle», nicht zuletzt deshalb hat der Beitrag (wie auch die anderen) eine offene Struktur mit eingestreuten, farblich abgesetzten Zitaten und Mikrogeschichten wie derjenigen aus Zermatt.

Der inhaltlich offene Stil ist Programm aller 33 beitragenden Historikerinnen und Historiker. Die Schweizergeschichte wird heute nicht mehr als eine lineare Erzählung, als eidgenössische Autobahn in die Gegenwart verstanden. Neben den klassischen historischen Dimensionen Herrschaft, Wirtschaft, Kultur und neuerdings Umwelt ermöglichen 22 «Fensterbeiträge» mehr Komplexität mit aktuellen Themen wie der Sprachenvielfalt, den Fremden in der Schweiz oder der Schweizer Armee.

Und trotzdem haben die Mikrogeschichten eine Verbindung zur «grossen Geschichte»: Die Zermatter hygienebedingte Epidemie passierte in einer Schweiz, deren sauberes und gesundes Image zum nationalen Selbstverständnis gehört. Die Mini-Katastrophe geschah am Matterhorn, dem Symbol des Alpentourismus, der wesentlich mithalf, die Schweiz zur starken, modernen Wirtschaftsnation zu machen. Die Epidemie spiegelte das gespaltene Verhältnis zu den Fremden in der Schweiz beispielhaft: die Offen- heit gegenüber Ausländern, wenn sie das Land als Touristen bereisen, und gleichzeitig die reflexartige Abwehr gegen sie, wenn sie als Saisonniers neben ihrer Arbeitskraft auch Probleme mit sich bringen. Deshalb macht es Sinn, wenn Georg Kreis die Zermatt-Vignette auf eine Seite platziert hat, in der es sonst um allgemeingeschichtliche Themen wie das Parteienspektrum, die Zauberformel, die «Überfremdungsdebatte» und die sich bereits damals anbahnenden politischen «68er»-Protestbewegungen geht.

Auch die anderen Kapitel weisen medizinhistorische Themen als Teil einer «grossen» Schweizer Geschichte aus. Mediziner und Gelehrte wie Rousseau, Scheuchzer oder Haller bastelten mit ihren Alpenforschungen an der Idee des starken und freien Schweizers als «Homo Alpinus». Die frühe, für die europäische Arbeitsmedizin bedeutsame Glarner Fabrikgesetzgebung entstand aus der bürgerlichen Tradition des sozialen Engagements. Die späte Einführung der Krankenversicherung können wir nur vor dem Hintergrund des schweizerischen Föderalismus verstehen. Im früh ermöglichten Medizinstudium der Frauen und im spät eingeführten Frauenstimmrecht spiegeln sich Widersprüche zwischen fortschrittlichen Beschlüssen einzelner männlicher Führungsgremien und einer vom Mehr der Schweizer Männer bestimmten Geschlechterpolitik.

Auch beim Typhus-Skandal im Oberwallis fand man übrigens einen sehr schweizerischen Weg. Nach einer Phase des Verschleierns und Verschweigens platzte die Bombe. Als erste Reaktion wurden die Saisonniers zu Sündenböcken gemacht. Das hochentwickelte Schweizer Gesundheitssystem konnte die Epidemie schnell beenden. Die Ursachen wurden mit grossem Aufwand untersucht. Aber wer für die Missstände eigentlich verantwortlich war, konnte nie eindeutig geklärt werden. Besser geklärt wurde in der Zukunft das Zermatter Abwasser [2]. Man wollte ja vor allem in die Zukunft sehen. Schon im Frühsommer hiess es, die Vorbestellungen für die kommende Wintersaison stünden besser denn je [3].

\section{Eberhard Wolff*}

1 Kreis G (Hrsg.). Die Geschichte der Schweiz. Basel: Schwabe; 2014. (645 Seiten, 372 Abb. 128 CHF ISBN 978-3-7965-2772-2).

2 Vouilloz Burnier MF. 1963. Typhus in Zermatt. Eine regionale Epidemie mit internationalen Folgen. Visp: Rotten Verlag; 2010.

3 Neue Zürcher Zeitung vom 2. März 1988, S. 9. 